\title{
A Prognostic Marker in Idiopathic Sudden Sensorineural Hearing Loss: Serum Calprotectin
}

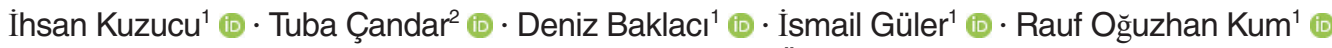 \\ Hande Arslan ${ }^{3}$ (i) $\cdot$ Müge Özcan ${ }^{1}$ (1)
}

${ }^{l}$ Department of Otorhinolaryngology, Ankara Numune Training and Research Hospital, Ankara; ${ }^{2}$ Department of Biochemistry, Ufuk University, Ankara; ${ }^{3}$ Department of Otorhinolaryngology, Dışkapı Yıldırım Beyazıt Training and Research Hospital, Ankara, Turkey

Objectives. Calprotectin, a protein released by neutrophils, has been used in many studies as a biomarker showing the presence of inflammation. In this study, it was aimed to investigate the relationship between serum calprotectin level and response to the treatment of idiopathic sudden sensorineural hearing loss (ISSHL).

Methods. The present study is a prospective, cross-sectional historical cohort study. The study group consisted of 44 patients with ISSHL, and the control group consisted of 41 healthy volunteers without ear pathology. At the same time, patients in the study group were divided into three groups according to the response to ISSHL treatment (recovered, partially recovered, unrecovered). The relationship between the groups was statistically evaluated in terms of serum calprotectin levels.

Results. The mean serum calprotectin value was $75.67 \pm 19.48 \mathrm{ng} / \mathrm{mL}$ in the study group and $50.24 \pm 29.14 \mathrm{ng} / \mathrm{mL}$ in the control group $(P=0.001)$. Serum calprotectin value according to the severity of hearing loss in the mild, moderate and severe was $66.20 \pm 8.82,70.35 \pm 16.77$, and $91.23 \pm 19.73 \mathrm{ng} / \mathrm{mL}$, respectively. Serum calprotectin value in the severe group was significantly higher compared to the moderate and mild groups ( $P=0.004, P=0.001$, respectively). Serum calprotectin value according to the treatment response in the recovered, partially recovered and unrecovered groups was $63.36 \pm 11.54,80.17 \pm 12.06$, and $85.33 \pm 22.33 \mathrm{ng} / \mathrm{mL}$, respectively. Serum calprotectin value in the recovered group was significantly lower compared to the partially recovered and unrecovered groups $(P=0.002$, $P=0.001$, respectively).

Conclusion. Serum calprotectin value informs the clinician about both the severity of hearing loss and the response to treatment. Hence, serum calprotectin can be used as an important biomarker in ISSHL patients for the determination of the prognosis of disease.

Keywords. Calprotectin; Idiopathic; Sensorineural Hearing Loss

\section{INTRODUCTION}

Idiopathic sudden sensorineural hearing loss (ISSHL) is characterized by a sensorineural hearing loss of at least $30 \mathrm{~dB}$ and at least three consecutive frequencies, which are noticed in 3 days

- Received March 6, 2019

Revised May 30, 2019

Accepted June 12, 2019

- Corresponding author: Deniz Baklacı

Department of Otorhinolaryngology, Ankara Numune Training and

Research Hospital, 931 Avenue 945 st, Cankaya,Ankara,Turkey

Tel: +90-05301757072, Fax: +90-3125044001

E-mail: doktorent@gmail.com or less [1]. Sudden hearing loss is one of the otological emergencies. Although the recovery rate is high, there may be partial or no improvement in some cases. In patients with hearing loss, social life and daily activities are negatively affected and patients experience social isolation and depression $[2,3]$.

Calprotectin, a member of the S100 protein family known as MRP8/MR14, S100A8/S100A9 and calgranulin, is one of the major antimicrobial human leukocyte protein. Calprotectin has bacteriostatic properties [4]. Depending on inflammation, calprotectin reaches the bloodstream through the neutrophil activation or epithelial adhesion of monocytes, so that the elevated calprotectin value in the serum can be used as a strong clinical

Copyright $\odot 2020$ by Korean Society of Otorhinolaryngology-Head and Neck Surgery

This is an open-access article distributed under the terms of the Creative Commons Attribution Non-Commercial License (https://creativecommons.org/licenses/by-nc/4.0)

which permits unrestricted non-commercial use, distribution, and reproduction in any medium, provided the original work is properly cited. 
marker indicating the presence of inflammation [5]. Studies have shown that calprotectin has a strong proinflammatory effect $[6,7]$. It has also been shown that calprotectin is also elevated in pathological tissue in the case of inflammation [8]. In many inflammatory and autoimmune diseases such as larynx, stomach, lung, endometrial cancers, the expression and release of calprotectin increase [4].

There is no serum biomarker to determine the prognosis of ISSHL. The aim of this study was to determine the serum calprotectin level in ISSHL patients and to investigate the role of serum calprotectin in determining the prognosis of ISSHL patients.

\section{MATERIALS AND METHODS}

Our study is a prospective, cross-sectional historical cohort study. Our study was approved by the local Ethics Committee and carried out in accordance with the ethical principles of the Declaration of Helsinki (project no. E-17-1634). In the study, patients with unilateral ISSHL were included in the study group, and healthy volunteers with normal bilateral hearing functions were included in the control group. Written informed consent was obtained from all participants.

Patients with any autoimmune disease, a history of acute or chronic infection or symptoms of infection, hypertension, conductive hearing loss, angina pectoris, myocardial infarction, diabetes mellitus, metabolic syndrome, chronic obstructive pulmonary disease, amyloidosis, chronic renal failure, obstructive sleep apnea, active smoking or otological diseases were excluded from the study. The control group consisted of healthy volunteers without ear pathology and with normal audiological findings who applied to our center's health board before application for employment presenting their health status to their employer. The control group did not have any systemic disorders as the study group.

The audiometric examinations were performed in quiet chambers by an Interacoustics AC-40 (Denmark) clinical audiometer according to the manufacturer's instructions, and all tests were performed by the same audiometrist. Audiological data were re-

\section{H I}

- Serum calprotectin levels were found higher in patients with idiopathic sudden sensorineural hearing loss (ISSHL) than healthy participants, and higher in patients with severe ISSHL than in other ISSHL patients.

- Serum calprotectin value was found to be higher in ISSHL patients who did not respond to treatment compared to other ISSHL patients.

- Serum calprotectin value seems to be a significant prognostic marker for the determination of response to treatment in ISSHL. ported according to the recommended methods of the Hearing Committee at the American Academy of Otolaryngology-Head and Neck Surgery, which has approved a new minimum standard for reporting hearing results in clinical studies [9]. Patients with ISSHL had at least $30 \mathrm{~dB}$ hearing loss at three consecutive frequencies, which was not associated with other known pathologies, including Meniere's disease, autoimmune disease, ototoxicity, or neoplasm developing within 72 hours of the onset of complaints. Hearing loss was unilateral in all patients.

All patients were treated with a standard ISSHL protocol containing an oral prednisone dose of $1 \mathrm{mg} / \mathrm{kg}$ per day (maximum dose $80 \mathrm{mg}$ ) the first 7 days, followed by gradual drug tapering in the next 7 days. Before the treatment, the patients with a hearing loss depending on ISSHL were divided into three classes as mild at a pure tone average (PTA) of less than $40 \mathrm{~dB}$, moderate between 40 and $80 \mathrm{~dB}$, and severe over $80 \mathrm{~dB}$ [10]. The response of the patients to the treatment was classified as follows (according to the recovery observed in a 1-month followup): (1) recovered: the posttreatment hearing loss value less than $10 \mathrm{~dB}$ compared to the initial hearing loss value or the intact ear PTA; (2) partially recovered: the posttreatment hearing loss value of more than $10 \mathrm{~dB}$ compared to the initial PTA or the intact ear PTA and recovery of more than $50 \%$; (3) unrecovered: the recovery less than $10 \mathrm{~dB}$ compared to the initial PTA [1].

Age, sex, serum calprotectin value, the neutrophil-to-lymphocyte ratio (NLR) and other laboratory data were recorded for all groups. Serum calprotectin levels and NLR values were also compared between the groups. At the same time, the relationship between serum calprotectin values was evaluated according to the severity of hearing loss and response to treatment for ISSHL patients.

Serum calprotectin value was measured in the venous blood taken from the patients before start of treatment to rule out an influence of treatment on the laboratory vales. Cytometry scattergrams were obtained within 2 hours of blood sampling. Calprotectin measurement was performed on the ChemWell 2900 (Awareness Technology, Palm City, FL, USA) enzyme-linked immunosorbent assay (ELISA) device using the Human CALP (calprotectin) ELISA kit. Results were reported in $\mathrm{ng} / \mathrm{mL}$. Intraassay and Interassay CV\% (coefficient variation) values are given as 3.92 and 4.76, respectively. All samples were analyzed in pairs, and the mean values were used for statistical values.

Statistical data analysis was performed using IBM SPSS ver. 21.0 (IBM Corp., Armonk, NY, USA). Data were expressed as mean \pm standard deviation (SD) for data with normal distribution, and median (minimum-maximum) for data without normal distribution. Shapiro-Wilk tests were used to check normality assumption for all parameters. Student $t$-test was used for independent samples to analyze serum calprotectin, and NLR value. The Mann-Whitney $U$-test was used to compare the groups without normal distribution. Correlations between the variable 
pairs were analyzed using the Pearson correlation test. A $P$-value $<0.05$ was accepted as statistically significant.

\section{RESULTS}

There were 44 patients in the study group (male: $n=23,52.3 \%$; female: $\mathrm{n}=21,47.7 \%$ ) and 41 volunteers in the control group (male: $n=20,48.8 \%$; female: $n=21,51.2 \%$ ). A total of 85 individuals were included in the study. The mean age of the study and control groups were $43.4 \pm 14.7$ years and $38.8 \pm 14.1$ years, respectively. There was no statistically significant difference between the groups in terms of sex and age $(P=0.726$ and $P=0.180$, respectively) (Table 1$)$.

The laboratory data of the study and control groups are presented in Table 1.The mean serum calprotectin value was $75.67 \pm$ $19.48 \mathrm{ng} / \mathrm{mL}$ in the study group and $50.24 \pm 29.14 \mathrm{ng} / \mathrm{mL}$ in the control group, and the difference was statistically significant $(P=0.001)$ (Table 1$)$. The mean NLR value was $3.44 \pm 2.54$ in the study group and $2.04 \pm 0.64$ in the control group $(P=0.002)$.

Serum calprotectin levels were found to be $66.20 \pm 8.82 \mathrm{ng} / \mathrm{mL}$ in patients with mild hearing loss and $70.35 \pm 16.77 \mathrm{ng} / \mathrm{mL}$ in patients with moderate hearing loss and $91.23 \pm 19.73 \mathrm{ng} / \mathrm{mL}$ in patients with severe hearing loss. Serum calprotectin levels were significantly higher in patients with severe hearing loss than those

Table 1. Intergroup comparison of age, sex, serum calprotectin and NLR values of the patients and the control group

\begin{tabular}{lccc}
\hline Variable & Patient $(n=44)$ & Control $(n=41)$ & $P$-value \\
\hline Age $(\mathrm{yr})$ & $40(18-65)$ & $38(18-60)$ & 0.180 \\
Sex & & & 0.726 \\
$\quad$ Female & $21(47.7)$ & $21(51.2)$ & \\
$\quad$ Male & $23(52.3)$ & $20(48.8)$ & \\
WBC $\left(10^{3} / \mathrm{U}\right)$ & $6.9(5.2-8.3)$ & $6.8(5.6-8.5)$ & 0.790 \\
Platelet $\left(10^{3} / \mathrm{U}\right)$ & $262.26 \pm 3.62$ & $268.61 \pm 55.53$ & 0.632 \\
Neutrophil $\left(10^{3} / \mathrm{U}\right)$ & $4.2(3.1-5.4)$ & $3.5(2.9-5.7)$ & 0.031 \\
Lymphocyte $\left(10^{3} / \mathrm{U}\right)$ & $1.9(1.5-2.6)$ & $2.1(1.5-2.3)$ & 0.284 \\
NLR & $3.44 \pm 2.54$ & $2.04 \pm 0.64$ & 0.002 \\
Calprotectin $(\mathrm{ng} / \mathrm{mL})$ & $75.67 \pm 19.48$ & $50.24 \pm 29.14$ & 0.001 \\
\hline
\end{tabular}

Values are presented as median (range), number (\%), or mean \pm standard deviation.

$\mathrm{NLR}$, neutrophil-to-lymphocyte ratio; WBC, white blood cell.

Table 2. Relationship between serum calprotectin levels and severity of hearing loss

\begin{tabular}{lcccl}
\hline Variable & $\begin{array}{c}\text { Mild } \\
(n=9)\end{array}$ & $\begin{array}{c}\text { Moderate } \\
(n=22)\end{array}$ & $\begin{array}{c}\text { Severe } \\
(n=13)\end{array}$ & $P$-value \\
\hline NLR & $2.09 \pm 0.90$ & $3.88 \pm 2.82$ & $3.03 \pm 2.19$ & 0.251 \\
Calprotectin $(\mathrm{ng} / \mathrm{mL})$ & $66.20 \pm 8.82$ & $70.35 \pm 16.77$ & $91.23 \pm 19.73$ & $\left.0.001^{\mathrm{a}}\right)$ \\
\hline
\end{tabular}

Values are presented as mean \pm standard deviation.

NLR, neutrophil-to-lymphocyte ratio.

a) Mild vs. moderate, $P=0.249$; moderate vs. severe, $P=0.004$; mild vs. severe, $P=0.001$. with moderate and mild hearing loss $(P=0.004 ; P=0.001$, respectively) (Table 2). NLR were found to be $2.09 \pm 0.90 \mathrm{ng} / \mathrm{mL}$ in patients with mild hearing loss and $3.88 \pm 2.82 \mathrm{ng} / \mathrm{mL}$ in patients with moderate hearing loss and $3.03 \pm 2.19 \mathrm{ng} / \mathrm{mL}$ in patients with severe hearing loss $(P=0.251)$ (Table 2).

The mean value of serum calprotectin in patients with ISSHL was $85.33 \pm 22.33$ in the unrecovered group, $80.17 \pm 12.06$ in the partially recovered group, and $63.36 \pm 11.54$ in the recovered group. The difference between the groups was statistically significant $(P=0.001)$ (Table 3$)$. The NLR in patients with ISSHL was $2.94 \pm 1.93$ in the unrecovered group, $3.89 \pm 2.69$ in the partially recovered group, and 3.66 \pm 3.08 in the recovered group. The difference between the groups was not statistically significant $(P=0.566)$ (Table 3$)$.

The serum calprotectin levels in patients with moderate hearing loss is increased by 4.0 units compared to those with mild hearing loss $(95 \%$ confidence interval $[\mathrm{CI}], 1.379$ to $6.621 ; P=$ 0.004). The serum calprotectin levels in patients with severe hearing loss is increased by 25.538 units compared to those with mild hearing loss (95\% CI, 11.666 to $28.411 ; P<0.001 ; \mathrm{R}^{2}=0.912$ ) (Table 4).

The serum calprotectin levels in patients with partial recovery is decreased by 4.812 units compared to unrecovered group ( $95 \%$ CI, -9.011 to $-0.612 ; P=0.026$ ). The serum calprotectin levels in patients with recovery hearing loss is decreased by 21.529 units

Table 3. Comparison of laboratory data between the unrecovered, partially recovered, and recovered patients

\begin{tabular}{lcccl}
\hline Variable & $\begin{array}{c}\text { Unrecovered } \\
(n=17)\end{array}$ & $\begin{array}{c}\text { Partially } \\
\text { recovered } \\
(n=10)\end{array}$ & $\begin{array}{c}\text { Recovered } \\
(n=17)\end{array}$ & $P$-value \\
\hline NLR & $2.94 \pm 1.93$ & $3.89 \pm 2.69$ & $3.66 \pm 3.08$ & 0.566 \\
Calprotectin $(\mathrm{ng} / \mathrm{mL})$ & $85.33 \pm 22.33$ & $80.17 \pm 12.06$ & $63.36 \pm 11.54$ & $0.001^{\mathrm{a})}$ \\
\hline
\end{tabular}

Values are presented as mean \pm standard deviation.

NLR, neutrophil-to-lymphocyte ratio.

a) Unrecovered vs. partially recovered, $P=0.436$; partially recovered vs. recovered, $P=0.002$; unrecovered vs. recovered, $P=0.001$.

Table 4. Results of regression analysis for the severity of hearing loss and serum calprotectin value

\begin{tabular}{lrrr}
\hline Severity of hearing loss & B (SE) & $95 \% \mathrm{Cl}$ & $P$-value \\
\hline Moderate & $4.0(1.298)$ & 1.379 to 6.621 & 0.004 \\
Severe & $25.538(1.423)$ & 11.666 to 28.411 & $<0.001$ \\
\hline
\end{tabular}

$\mathrm{B}$, unstandardized beta; $\mathrm{SE}$, standard error; $\mathrm{Cl}$, confidence interval.

Table 5. Result of regression analysis for the response to treatment and calprotectin value

\begin{tabular}{lccr}
\hline Calprotektin & B (SE) & $95 \% ~ C l$ & $P$-value \\
\hline Partial recovery & $-4.812(2.079)$ & -9.011 to -0.612 & 0.026 \\
Recovery & $-21.529(1.790)$ & -25.144 to -17.915 & $<0.001$ \\
\hline
\end{tabular}

$\mathrm{B}$, unstandardized beta; $\mathrm{SE}$, standard error; $\mathrm{Cl}$, confidence interval. 
compared to unrecovered group (95\% CI, -25.144 to -17.915 ; $P<0.001 ; \mathrm{R}^{2}=0.780$ ) (Table 5).

\section{DISCUSSION}

Serum calprotectin levels were found higher in patients with ISSHL than healthy participants, and higher in patients with severe ISSHL than in other ISSHL patients. Serum calprotectin value was found to be higher in ISSHL patients who did not respond to treatment compared to other ISSHL patients.

Calprotectin proteins account for about $45 \%$ of all cytosolic proteins in neutrophils and about $5 \%$ of all monocyte/macrophage proteins [8]. Calprotectin proteins have been reported to interact with heparan sulfate proteoglycans such as perlecan, agrin and collagen XVIII on extracellular matrix components. When monocytes and neutrophils adhere to the vascular endothelium, they release S100A8/A9 proteins to the endothelium [8]. Studies on calprotectin show that it is a biomarker that can be used for the early diagnosis of various cancers $[4,11]$. However, it is thought that the concentration of calprotectin increases due to the inflammatory response in cancerous tissues [4]. In addition, calprotectin inhibits intracellular enzymes involved in cellular proliferation and creates an apoptosis-inducing effect. Calprotectin also inhibits matrix metalloproteinase, which plays a role in the growth and metastasis of cancer cells [12].

In the study of Masuda et al. [13], it was reported that neutrophil and inflammatory marker levels were higher in SHL patients than the healthy population. In another study, SHL patients were classified as mild ( $<40 \mathrm{~dB}$ loss for any frequency), moderate $(40-80 \mathrm{~dB})$ and severe $(>80 \mathrm{~dB})$ according to the severity of hearing loss, and the mean NLR values in SHL patients were compared with healthy individuals. The mean NLR values were found to be significantly higher in patients with severe sensorineural hearing loss than in healthy individuals $[10,14]$. In our study, we found that NLR is insufficient to determine the severity of ISSHL. We divided the severity of ISSHL into three groups as mild, moderate and severe, and we investigated the relationship between calprotectin, the severity of disease and the healthy volunteer control group. As a result of the present study, we found a statistically significant difference in calprotectin value between the control group and the patient group. We also observed that serum calprotectin levels were significantly higher in patients with severe hearing loss compared to patients with moderate and mild hearing loss and there was a significant correlation between severity of disease and serum calprotectin values. Unlike NLR, serum calprotectin value can be used to determine the severity of ISSHL biomarker. In our study, we used the more common classification in meta-analysis studies and the latest treatment guidelines to evaluate the recovery criteria $[1,15]$ and found that serum calprotectin value in the unrecovered group was significantly higher compared to the partially recovery and recovery groups. Hence, we think that serum calprotectin value is a biomarker that can be used to determine the response of ISSHL patients to treatment. In this way, prognosis of disease can be estimated before starting treatment, and other treatment options, such as intratympanic injection or hyperbaric oxygen therapy, can be combined with steroid treatment in patients with high pretreatment calprotectin values.

Hearing impaired individuals often have difficulty in hearing background noise and identifying sound sources. It was reported that the incidence of severe depressive disorder is higher in the hearing impaired patients than in the general population [16]. Consistently with this hypothesis, depressive disorder is more common in patients who do not recover after ISSHL [3]. The current evidence suggests that psychological disorders such as depression and anxiety have a negative effect on the quality of life of patients with SSHL [3]. The authors of this study reported that clinicians increased the risk of ISSHL to develop affective disorders in patients, suggesting that special measures should be taken to reduce the risk of depression and anxiety disorder [3]. In our study, we planned to use serum calprotectin as a biomarker in order to detect patients with worse prognosis earlier during the onset of hearing loss. At the end of our study, we found that serum calprotectin is correlated with the severity of ISSHL. In patients with ISSHL, it is possible to estimate the response of the disease to treatment by examining the serum calprotectin value obtained before treatment, in this way we can inform patients about the prognosis of disease.

Our study suggests that serum calprotectin value informs the clinician about both the severity of ISSHL disease and the response to ISSHL treatment. Serum calprotectin can be used as an important prognostic biomarker in ISSHL patients for the estimation of response to treatment. This issue should be investigated on more patients in a multicenter study.

\section{CONFLICT OF INTEREST}

No potential conflict of interest relevant to this article was reported.

\section{ORCID}

$\begin{array}{ll}\text { İhsan Kuzucu } & \text { https://orcid.org/0000-0001-5773-4126 } \\ \text { Tuba Çandar } & \text { https://orcid.org/0000-0002-3922-5915 } \\ \text { Deniz Baklac1 } & \text { https://orcid.org/0000-0001-8449-4965 } \\ \text { İsmail Güler } & \text { https://orcid.org/0000-0001-6093-6757 } \\ \text { Rauf Oğuzhan Kum } & \text { https://orcid.org/0000-0002-9639-0204 } \\ \text { Hande Arslan } & \text { https://orcid.org/0000-0003-0344-2712 } \\ \text { Müge Özcan } & \text { https://orcid.org/0000-0003-2384-3564 }\end{array}$ 


\section{AUTHOR CONTRIBUTIONS}

Conceptualization: İK, TÇ, DB. Data curation: İK, TÇ, HA. Formal analysis: DB, IG, ROK, HA. Methodology: IK, MÖ. Project administration: IK, DB, MÖ. Visualization: TÇ, IG, HA. Writing original draft: IK, DB, İG.Writing - review\&editing: ROK, MÖ.

\section{REFERENCES}

1. Stachler RJ, Chandrasekhar SS, Archer SM, Rosenfeld RM, Schwartz SR, Barrs DM, et al. Clinical practice guideline: sudden hearing loss. Otolaryngol Head Neck Surg. 2012 Mar;146(3 Suppl):S1-35.

2. Sano H, Okamoto M, Ohhashi K, Iwasaki S, Ogawa K. Quality of life reported by patients with idiopathic sudden sensorineural hearing loss. Otol Neurotol. 2013 Jan;34(1):36-40.

3. Kim JY, Lee JW, Kim M, Kim MJ, Kim DK. Association of idiopathic sudden sensorineural hearing loss with affective disorders. JAMA Otolaryngol Head Neck Surg. 2018 Jul;144(7):614-21.

4. Topuz MF, Binnetoglu A, Yumusakhuylu AC, Sari M, Baglam T, Gerin F. Circulating calprotectin as a biomarker of laryngeal carcinoma. Eur Arch Otorhinolaryngol. 2017 Jun;274(6):2499-504.

5. Striz I, Trebichavsky I. Calprotectin: a pleiotropic molecule in acute and chronic inflammation. Physiol Res. 2004;53(3):245-53.

6. Vogl T, Tenbrock K, Ludwig S, Leukert N, Ehrhardt C, van Zoelen MA, et al. Mrp8 and Mrp14 are endogenous activators of Toll-like receptor 4, promoting lethal, endotoxin-induced shock. Nat Med. 2007 Sep;13(9):1042-9.

7. Loser K, VoglT, Voskort M, Lueken A, Kupas V, Nacken W, et al. The Toll-like receptor 4 ligands Mrp8 and Mrp14 are crucial in the de- velopment of autoreactive CD8+ T cells. Nat Med. 2010 Jun;16(6): 713-7.

8. Van Crombruggen K,VoglT, Perez-Novo C, Holtappels G, Bachert C. Differential release and deposition of S100A8/A9 proteins in inflamed upper airway tissue. Eur Respir J. 2016 Jan;47(1):264-74.

9. Gurgel RK, Jackler RK, Dobie RA, Popelka GR. A new standardized format for reporting hearing outcome in clinical trials. Otolaryngol Head Neck Surg. 2012 Nov;147(5):803-7.

10. Kum RO, Ozcan M, Baklaci D, Yurtsever Kum N, Yilmaz YF, Unal A, et al. Investigation of neutrophil-to-lymphocyte ratio and mean platelet volume in sudden hearing loss. Braz J Otorhinolaryngol. 2015 Nov-Dec;81(6):636-41.

11. Ni Bhriain H, Trovik J, Wik E, Stefansson IM, Akslen LA, Salvesen $\mathrm{HB}$, et al. Plasma calprotectin concentrations in women with endometrial carcinoma. Gynecol Oncol. 2009 Sep;114(3):491-5.

12. Yui S, Nakatani Y, Mikami M. Calprotectin (S100A8/S100A9), an inflammatory protein complex from neutrophils with a broad apoptosis-inducing activity. Biol Pharm Bull. 2003 Jun;26(6):753-60.

13. Masuda M, Kanzaki S, Minami S, Kikuchi J, Kanzaki J, Sato H, et al. Correlations of inflammatory biomarkers with the onset and prognosis of idiopathic sudden sensorineural hearing loss. Otol Neurotol. 2012 Sep;33(7):1142-50.

14. Ozler GS. Increased neutrophil-lymphocyte ratio in patients with idiopathic sudden sensorineural hearing loss. J Craniofac Surg. 2014 May;25(3):e260-3.

15. Conlin AE, Parnes LS. Treatment of sudden sensorineural hearing loss. II. A meta-analysis. Arch Otolaryngol Head Neck Surg. 2007 Jun;133(6):582-6.

16. Lee AT, Tong MC, Yuen KC, Tang PS, Vanhasselt CA. Hearing impairment and depressive symptoms in an older chinese population. J Otolaryngol Head Neck Surg. 2010 Oct;39(5):498-503. 